This item was submitted to Loughborough's Research Repository by the author.

Items in Figshare are protected by copyright, with all rights reserved, unless otherwise indicated.

\title{
Reliability of sequential systems using the cause-consequence diagram method
}

PLEASE CITE THE PUBLISHED VERSION

PUBLISHER

(C) IMechE / Professional Engineering Publishing

LICENCE

CC BY-NC-ND 4.0

REPOSITORY RECORD

Andrews, J.D., and L.M. Ridley. 2008. "Reliability of Sequential Systems Using the Cause-consequence Diagram Method”. figshare. https://hdl.handle.net/2134/3815. 
This item was submitted to Loughborough's Institutional Repository (https://dspace.lboro.ac.uk/) by the author and is made available under the following Creative Commons Licence conditions.

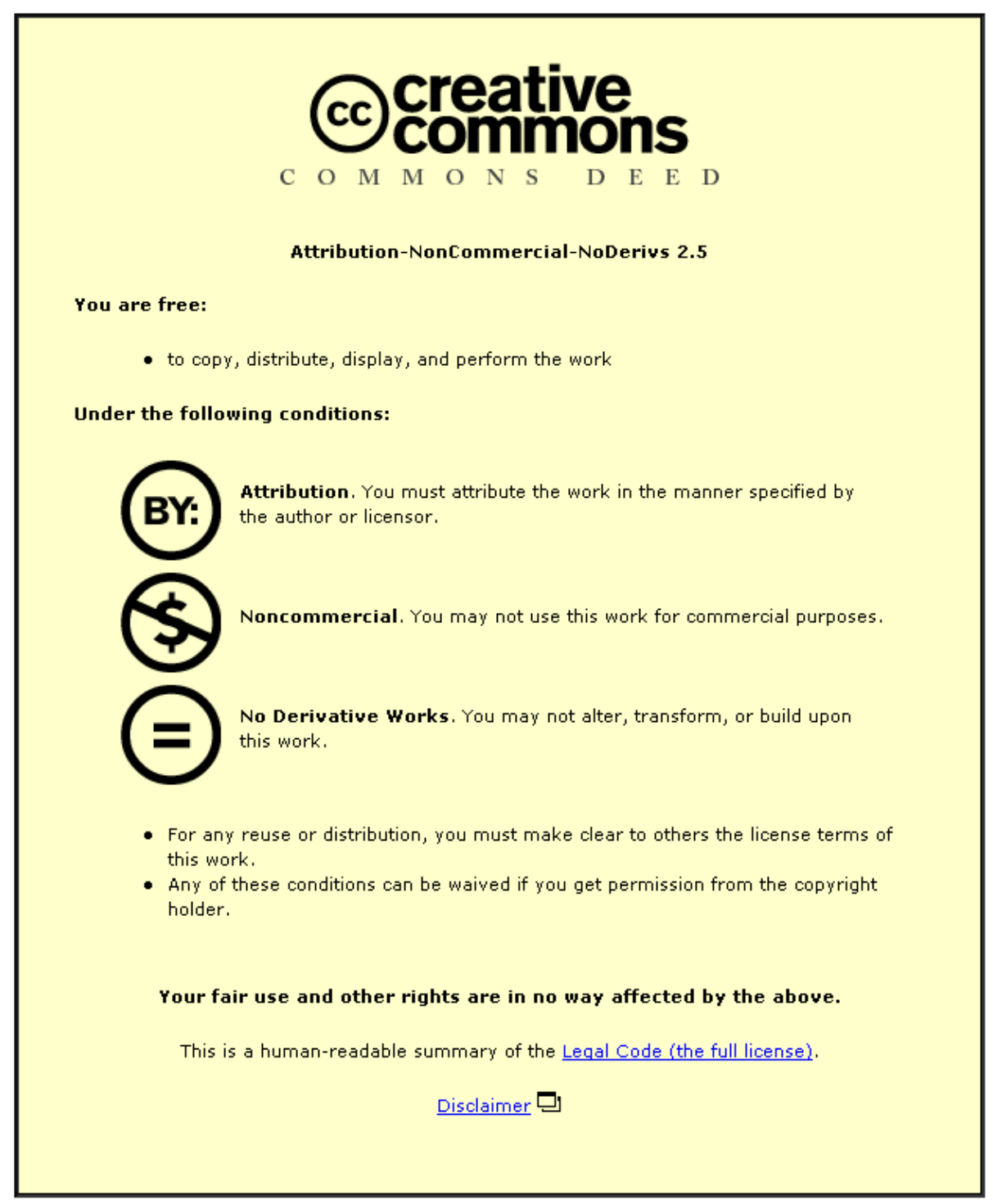

For the full text of this licence, please go to: http://creativecommons.org/licenses/by-nc-nd/2.5/ 


\title{
Reliability of sequential systems using the cause-consequence diagram method
}

\author{
J D Andrews and L M Ridley* \\ Department of Mathematical Sciences, Engineering Mathematics Division, Loughborough University, Leicestershire, \\ UK
}

\begin{abstract}
In many industrial systems, where safety is of the utmost importance, it is necessary that expedient tools for accident analysis are available and employed at the design stage. Such tools must be able to handle large systems in a systematic way and display the factors that are of vital importance for the functionality of the system. The technique of fault tree analysis (FTA) is commonly used to assess the failure probability of such systems. The fault tree represents the failure logic of the system in an inverted tree structure and has the advantage that it provides very good documentation of the way the failure logic was developed. Conventional fault tree quantification requires a number of assumptions regarding the system. One of these is that the basic events in the tree occur independently. This condition is not satisfied when sequential failures are encountered. Employing alternative methods, such as Markov methods, can result in the loss of the documentation that represents the failure logic of the system.

The cause-consequence diagram method is a tool that, like fault tree analysis, documents the failure logic but has the extra capability enabling the analysis of systems subject to sequential failures. In addition, the cause-consequence diagram identifies the complete set of system responses to any given initiating event.

This paper is concerned with the cause-consequence diagram method and its application to sequentially operating systems. It extends previous work by providing more rigorous guidelines to enable the construction of the diagram and an analysis methodology that can be used when dependencies exist between the events featured in the decision boxes. A new symbol distinguishing between events that exist at a specified point in time and those that occur at that time is introduced to facilitate the analysis.
\end{abstract}

Keywords: cause-consequence analysis, fault tree analysis, dependencies

\section{INTRODUCTION}

When investigating potential accident sequences, the time between the occurrences of events can be an important parameter [1]. This type of system could be characterized as one with various shutdown mechanisms that are initiated given the presence of some initiating event, e.g. when a pressure limit is exceeded. In order to identify all relevant accidents for a such a system, Nielsen stated that the safety assessment tool used must be able to determine the possible causes of the accident

The MS was received on 15 June 2000 and was accepted after revision for publication on 17 May 2001.

*Corresponding author: Department of Mathematical Sciences, Engineering Mathematics Division, Loughborough University, Loughborough, Leicestershire LE11 3TU, UK. event and identify the possible consequences given that one or more of the accident limiting provisions could fail.

The technique of fault tree analysis (FTA) [2] is commonly used to assess the probability of failure of industrial systems. This method represents the failure logic of the system in an inverted tree structure and provides very good documentation of the way that the system failure logic was developed. The FTA technique, however, is incapable of identifying both the possible causes of an undesirable event AND all the possible consequences resulting from it. In addition to this, the FTA method cannot accurately analyse systems containing sequential failures. Markov models [3] do not require the assumption of independence, as with the FTA method, and can therefore be used accurately to analyse sequential failures. This modelling technique describes the system in a state transition diagram. The state 
transition diagram is not as easy to construct as a fault tree and contains no textual description regarding the failure logic of the system. As with the FTA method, the Markov analysis method has the ability to identify the possible causes of the undesirable events yet is incapable of identifying all the possible consequences resulting from it.

A technique has been developed that possesses the ability to identify the causes of an undesired event and from this event develop all possible system consequences. The technique is known as the causeconsequence diagram method. The cause-consequence diagram method was developed at RISO National Laboratories, Denmark, in the 1970s, specifically to aid in the reliability and risk analysis of nuclear power plants in Scandinavian countries [4]. The method was created to assist in the cause-consequence accident analysis of the nuclear plants, which involved identification of the potential modes of failure of individual components and then relating these causes to the ultimate consequences for the system [5]. The method can be seen as superior to event tree analysis (ETA) [4], which is also capable of identifying all consequences of a given critical event, as it models at component level and therefore is functionality driven and not subsystem driven. In addition to this, the cause-consequence diagram method can account for time delays, which is not a feature available in the ETA method. Nielsen stated that, as well as being a tool for illustrating the consequences of particular failures, the method could also serve as a basis from which the probability of occurrence of the individual consequences could be evaluated. The consequences evaluated include those that illustrate the system functioning as intended and those that illustrate an undesirable failure sequence. Several authors have used the technique as the main analysis tool for a safety assessment [6-9]. However, the documentation of the quantification of the cause-consequence diagram is limited and a generalized analysis method or even rigorous definitions of the meaning of the symbols to enable quantification is yet to be developed. This is the subject considered in this paper. Rules for construction and quantification of the cause-consequence diagram have been developed and applied to an industrial system.

The quantification method developed can be automated for computerized system assessment and has the capability to deal with dependencies that can occur when analysing systems whose operation is sequential. These dependencies include component failures that are repeated as causes of more than one decision box event. The occurrence of such a common event therefore influences the outcome of more than one of the decision boxes in any path. A second class of dependent events encompasses those events whose failures are inconsistent, and the occurrence of a specific component failure mode excludes the possibilities of other components failing, which feature in other parts of the diagram and are therefore mutually exclusive.

\section{CAUSE-CONSEQUENCE DIAGRAM METHOD}

The main principle of the cause-consequence diagram technique is based on the occurrence of a critical event, i.e. an event that disturbs the balance of the process plant. The identification of the critical event is problem dependent, and choosing the correct place to start is important as there are very many possible initial events, not all of which have serious consequences. Focus should therefore only be made on functional failures of process components that directly affect the plant balance. Once a critical event has been identified, all relevant causes of the critical event and potential consequences are developed using two conventional reliability analysis methods. This situation is represented in Fig. 1.

The two reliability analysis tools used in the development of the cause-consequence diagram method are the FTA method and the ETA method. The FTA method is used in two independent situations to describe the causes of an undesired event. Firstly, the technique is used to describe the causes of the critical event. The second function for the fault tree method is to describe the failure causes of the accident-limiting systems (emergency shutdown systems). The event tree method is used as the link between the causes of the critical event and the various consequences that could result. The method is used to identify the various paths that the system could take, following the critical event, depending on whether certain subsystems/components function correctly or not. The relationship between the two reliability methods is shown in Fig. 2.

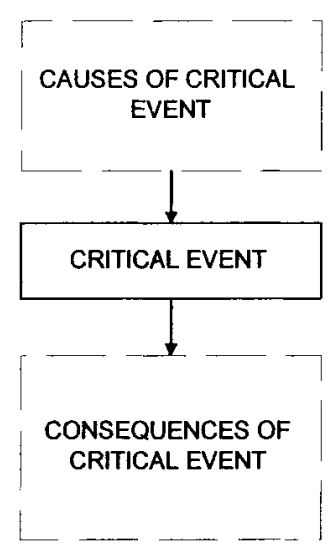

Fig. 1 Simple representation of a cause-consequence diagram structure 


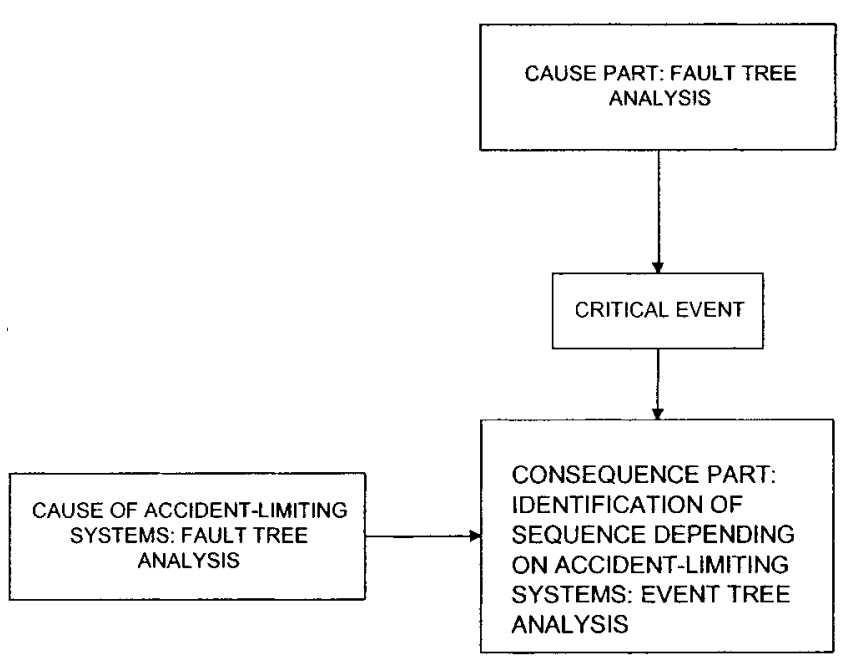

Fig. 2 Basic structure of a cause-consequence diagram

\section{SYMBOLS FOR CONSTRUCTION}

The symbols used for the construction of a causeconsequence diagram are depicted in Table 1. The overall structure of the cause-consequence diagram method is depicted in Fig. 3.

\section{RULES FOR CONSTRUCTION}

Rules for the construction of a cause-consequence diagram can be detailed in two separate sections, those for the cause part of the diagram and those for the consequence part of the diagram. For the cause part it should be noted that the rules postulated are those used in the construction of a fault tree structure. The rules for the construction of the cause diagram can be summarized as a three-step procedure:

1. Identification of the top event. The construction of the cause diagram begins with the definition of the undesired event, i.e. the system failure of interest.

2. Cause diagram development. Using a deductive process, the causes of the undesired event are discovered and connected by means of logical gates. The procedure is repeated until all events have been fully developed, i.e. the basic events are reached.

3. Validation of the diagram. For each gate used, all inputs must be both necessary and sufficient to produce the output event.

Similarly, a set of rules were devised for the construction of the consequence diagram:

1. Component ordering. The first step of the causeconsequence diagram construction is deciding on the order in which component failure events are to be taken. To ensure a logical development of the causes of the system failure mode, it was decided that the ordering should follow the temporal action of the system, for example the system activation for the function required given an initial critical event.

2. Consequence diagram development. The second stage involves the actual construction of the diagram. Starting from the initiating component, the functionality of each component or subsystem is investigated and the consequences of these sequences determined. If the decision box is governed by a subsystem, then the probability of failure will be obtained via a fault tree diagram.

3. Reduction. If any decision boxes are deemed irrelevant, for example the boxes attached to the NO and YES branches are identical and their outcomes and consequences are the same, then these should be removed and the diagram reduced to a minimal form. Removal of these boxes will in no way affect the end result.

\section{RULES FOR QUANTIFICATION}

The procedure for analysing an independent system modelled using a cause-consequence diagram begins with the assignment of probabilities/frequencies to each outlet branch stemming from a decision box. Following this, the probability of any one sequence is obtained by multiplication of the probabilities associated with each decision box in that sequence $[\mathbf{1 0}]$. The probability of any particular consequence is then obtained by the summation of the probability of each sequence that terminates in that consequence. This procedure, however, cannot be employed unless the failures of each decision box in a sequence are independent. Dependencies can exist in the cause-consequence diagram, and these must be dealt with prior to the quantification of the diagram.

\subsection{Rules for dependent failure events}

\subsubsection{Common failure events}

The first dependency that can arise is that the same failure event exists in more than one fault tree structure on the same path in the cause-consequence diagram. In order to deal with a common failure event, the event is extracted from the fault tree structures and placed in a new decision box preceding the first decision box that contains the common failure event. The original cause-consequence diagram is then duplicated on each outlet branch stemming from the new decision box. Following the NO outlet branch of the new decision box, the failure event is set to TRUE in any fault tree structure in which it is found. Similarly, following the YES outlet branch, the 
Table 1 Symbols used for construction of a cause-consequence diagram

Symbols for cause diagram
AND GATE:
Allows causality to pass up through the tree if at any time all inputs to the gate occur
OR GATE:
Allows causality to pass up through the tree if at any time at least one input to the gate occurs

\section{Symbol for consequence diagram Function description}

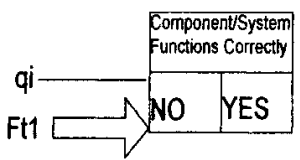

Ft1
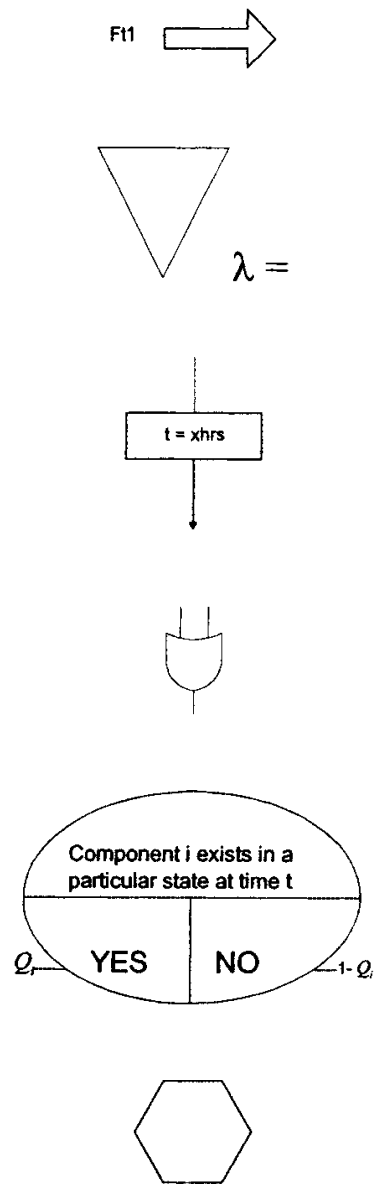

The decision box represents the functionality of a component/system. The NO box represents failure to perform correctly, the probability of which is obtained via a fault tree or single component failure probability $q_{i}$

Fault tree arrow represents the number of the fault tree structure that corresponds to the decision box

The initiator triangle represents the initiating event for a sequence where $\lambda$ indicates the rate of occurrence

Time delay 1 indicates that the time starts from the time at which the delay symbol is entered and continues up to the end of the time interval in the delay symbol

OR gate symbol:

Used to simplify the cause-consequence diagram when more than one decision box enters the same decision box or consequence box

Existence decision box represents a component existing in a certain state

Consequence box represents the outcome event due to a particular sequence of events 


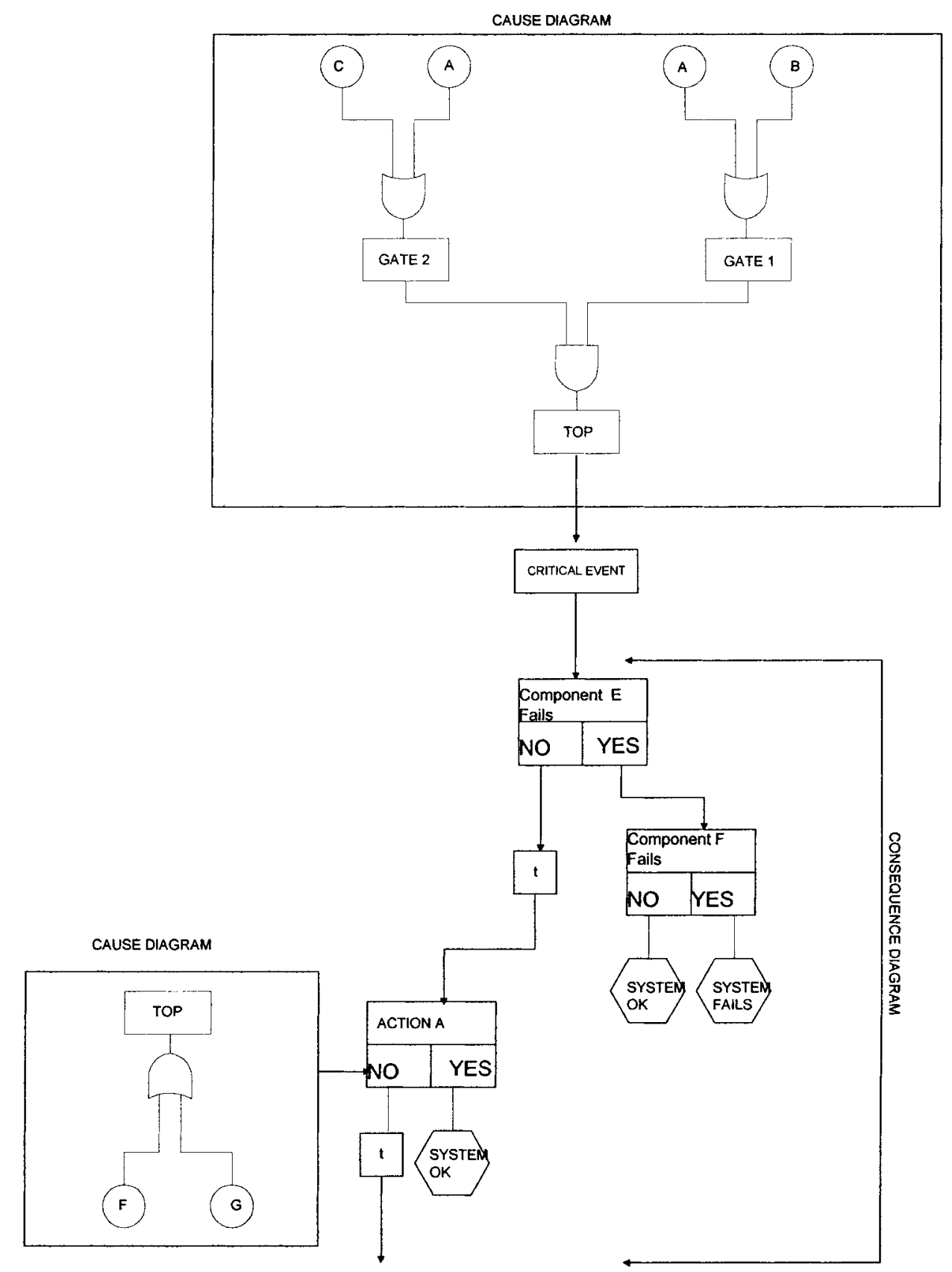

Fig. 3 Cause-consequence diagram structure

probability of failure of the common failure event is set to FALSE in any fault tree structure in which it is present.

\subsubsection{Inconsistent failure events}

In certain systems, components are required to perform different functions which, if successfully accomplished, results in the components residing in different states at different times. For example, initially a valve may be required to be closed and later in the sequence be open. For systems that are not in continuous operation, certain component failures could occur between operations. For example, the valve could fail between operations, which would be the cause of the valve being closed at the start of the next sequence, and later in the sequence the valve would be unable to open. To illustrate this, the simple cause-consequence diagram section shown in Fig. 4 can be utilized with the relevant fault trees depicted in Fig. 5.

In this example, the component $\mathrm{K} 2$ is required to perform two different functions; firstly to close-decision box 1 , and then, later in the sequence, to open-decision box 3. In order to model this type of failure accurately, the cause-consequence diagram requires modification 


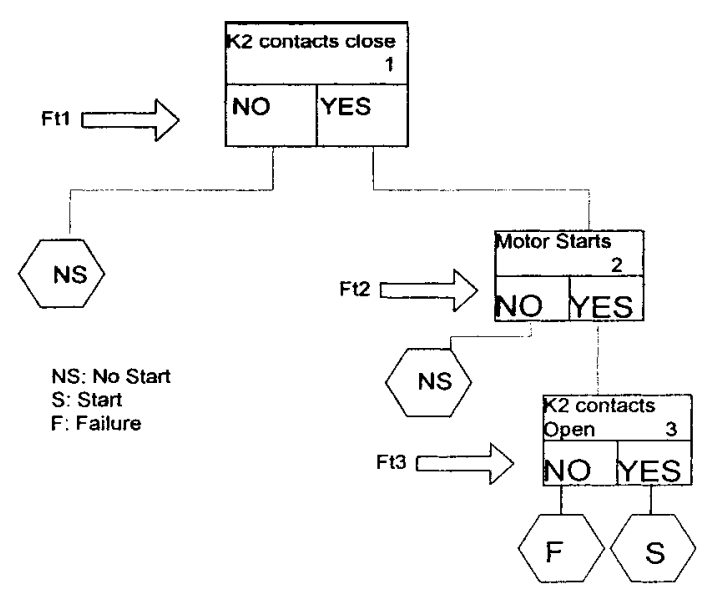

Fig. 4 Example cause-consequence diagram

prior to quantification. Employing a basic event labelling convention in a fault tree structure can be used to identify an inconsistent failure event. If two labels are the same apart from the last character, then they are deemed as inconsistent failure events and the first failure event represents the decision box containing the first failure mode and the second failure event represents the decision box containing the second failure mode. This can be seen for the cause-consequence diagram in Fig. 4, where Ft1 contains the basic event $\mathrm{K} 2 \mathrm{CO}$, the first failure mode, and $\mathrm{Ft} 3$ contains the basic event $\mathrm{K} 2 \mathrm{CC}$, the second failure mode. Following the identification of an inconsistent failure event, the second failure mode is inspected and, depending on whether the second failure mode is an unrevealed or revealed failure event, the cause-consequence diagram is different. If the second failure mode is a revealed failure, then it cannot fail between operations and be undetected. Therefore, the time to failure of the second failure mode is set equal to the time it takes the system to travel from the first failure event to the second failure event. If, on the other hand, the second failure mode is unrevealed, then it can occur between operations and be undetected. When this situation occurs, the second failure mode is extracted and placed in an existence decision box preceding the first failure event. The causeconsequence diagram is then duplicated on both outlet branches and, following the YES outlet branch of the existence decision box, the decision box containing the first failure mode is governed by the failure of the second failure mode. The second failure mode probability is set equal to 1 in all decision boxes beneath the existence decision box, and the first failure mode is set equal to 0 . Therefore, for Fig. 4, assuming $\mathrm{K} 2 \mathrm{CC}$ is an unrevealed failure event, the cause-consequence diagram illustrated in Fig. 6 would be created and reduced to the form shown in Fig. 7.

Following the NO outlet branch of the existence decision box results in the same scenario as if the failure had in fact been a revealed failure. Therefore, the second failure event occurs in the time it takes the system to travel from the first failure event to the second failure event.

Following the inspection of each sequence path in the cause-consequence diagram, and modification due to any identified dependent failure events, the cause-consequence diagram can be quantified by multiplying the probability associated with each decision box in each sequence. The probability of any consequence is then obtained via the summation of the probability of any sequence that terminates in that consequence.

\section{PRESSURE TANK SYSTEM}

The system used to illustrate the construction of a causeconsequence diagram is a pressure tank system that contains a start-up, shutdown sequence in addition to its operational phase [11]. The system configuration is given in Fig. 8 and the component individual functions and failure modes are represented in Table 2.

Initially, the system is considered to be in a dormant state and therefore de-energized. Switch $\mathrm{S} 1$ and relay contacts $\mathrm{K} 1$ and $\mathrm{K} 2$ are all open when in the dormant state, and the timer and pressure switch contacts are
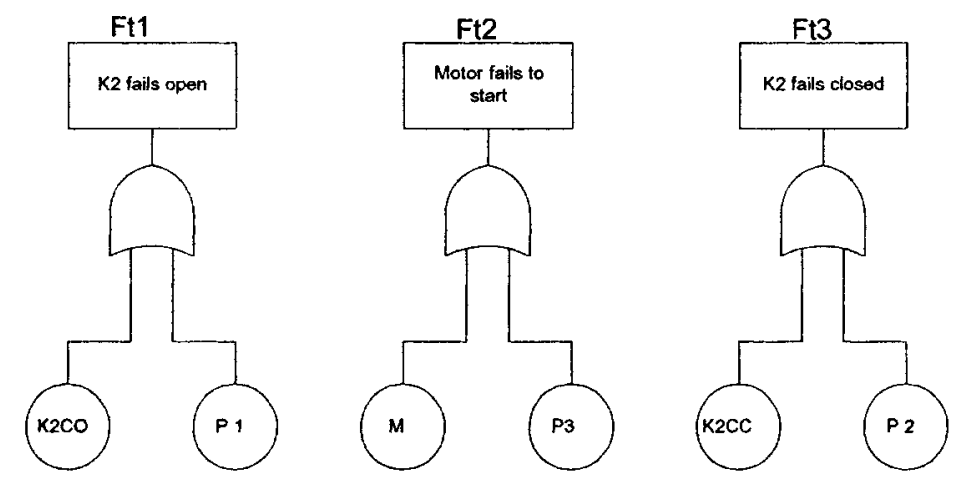

Fig. 5 Fault trees for the example cause-consequence diagram 


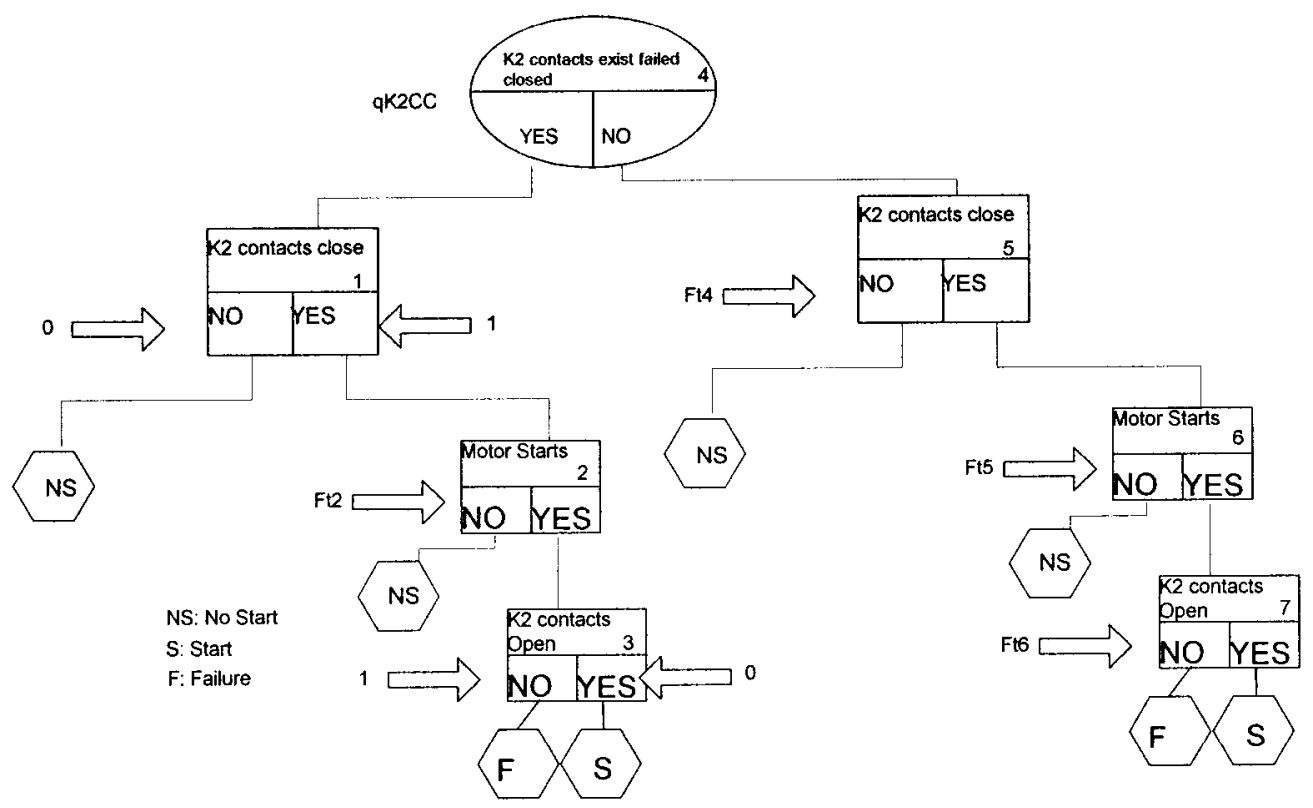

Fig. 6 Modified cause-consequence diagram for inconsistent failure modes

closed. Depressing switch S1 provides power to the coil of $\mathrm{K} 1$ which results in the closure of the $\mathrm{K} 1$ contacts. Relay K1 self-latches when $\mathrm{S} 1$ opens when released, and power is also supplied to $\mathrm{K} 2$, resulting in $\mathrm{K} 2$ contacts closing, which starts the pump motor. It is assumed that the tank takes $30 \mathrm{~min}$ to fill, and once the pressure threshold is reached the pressure switch contacts open, de-energizing $\mathrm{K} 2$, which results in the removal of power from the pump motor. After a period of time the tank becomes empty and the pressure switch closes, which energizes K2. The pump restarts and the filling process commences again. The tank is filled twice daily and the system is inspected at 6 monthly intervals for dormant failures.

In the event of the pressure switch failing to open, a safety feature is included in the form of the timer relay. Power is applied to the timer relay following the closure of the $\mathrm{K} 1$ contacts, which initiates a clock. If the clock registers $30 \mathrm{~min}$ of continuous pumping, then the timer relay contacts are opened which results in a break in the circuit to $\mathrm{K} 1$ and system shutdown.

The rules developed for the construction of a cause-

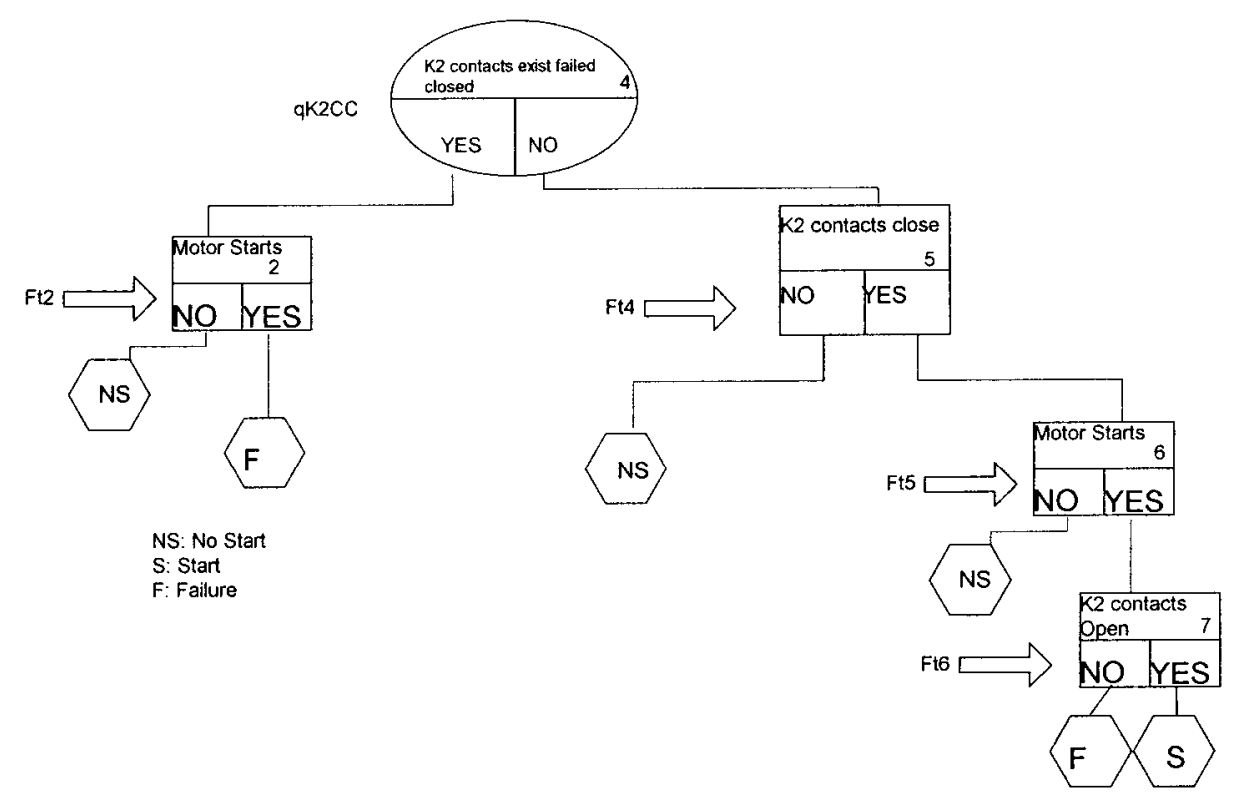

Fig. 7 Reduced cause-consequence diagram for inconsistent failure modes 


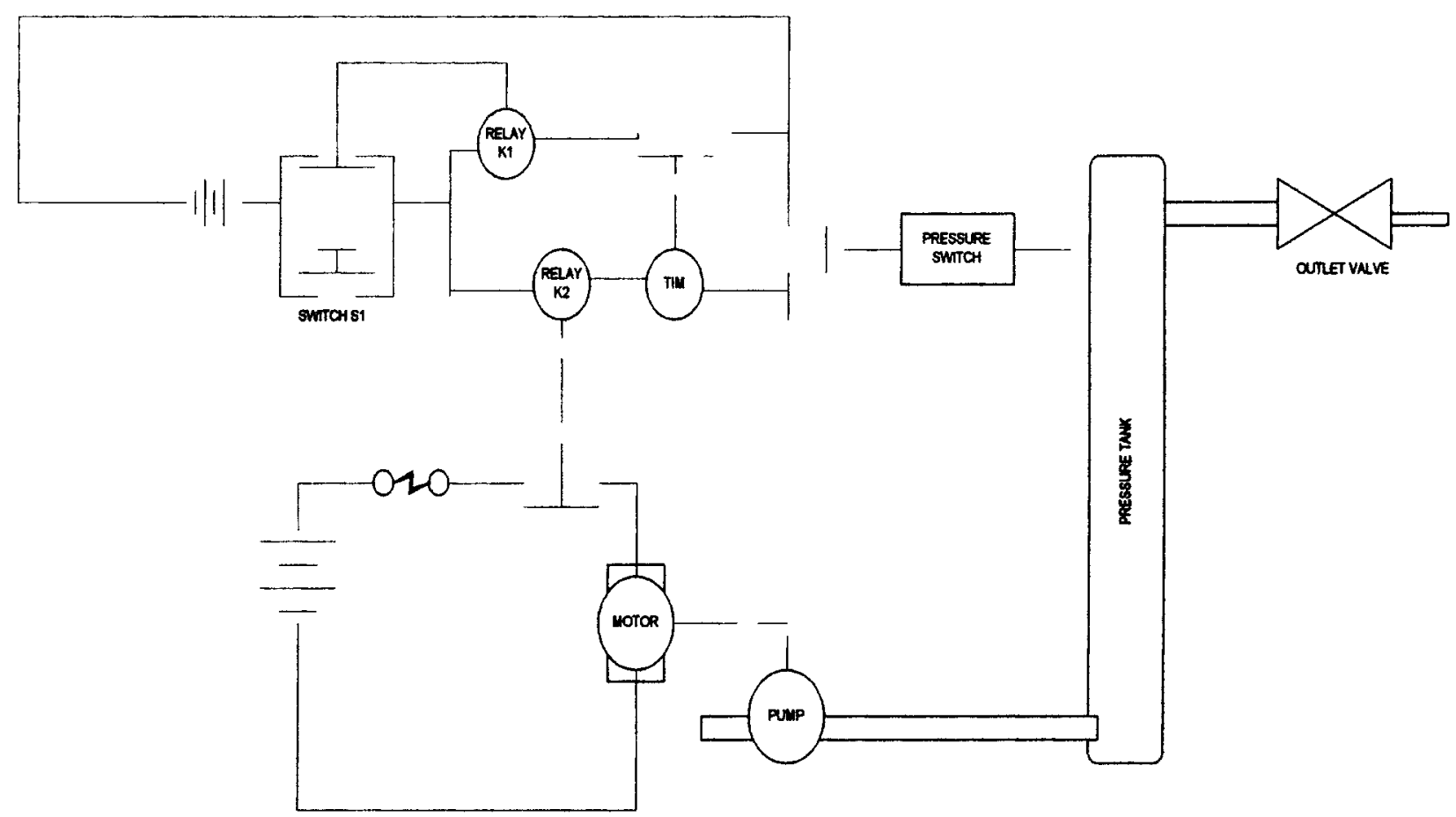

Fig. 8 Pressure tank system

consequence diagram were used to construct the causeconsequence diagram for the pressure tank system:

Step 1. Component failure event ordering. The ordering of the components for the construction of the causeconsequence diagram is selected by considering the temporal patterns of the system. For the pressure tank system, switch S1 is depressed, followed by its opening. Relay K1 energizes and powers K2 which powers the pump. Following $30 \mathrm{~min}$ of operation, the pressure switch should open. In the event that the pressure switch fails to open, the timer should time out and the timer contacts open. Given that the pressure switch opens, K2 contacts should de-energize, remov-

Table 2 Component functions and failure modes

\begin{tabular}{|c|c|c|c|c|}
\hline Component & Function & Failure modes & Effect on system & Failure type \\
\hline Switch S1 & To apply power to coil of relay $\mathrm{K} 1$ & S1C: Switch failed closed & $\begin{array}{l}\text { Circuit remains energized but can } \\
\text { be broken by } \mathrm{K} 2\end{array}$ & Unrevealed \\
\hline \multirow[t]{3}{*}{ Relay K1 } & \multirow[t]{3}{*}{$\begin{array}{l}\text { Electrically self-latched, applying } \\
\text { power to relay } \mathrm{K} 2\end{array}$} & K1D: Relay fails de-energized & No power to circuit & Revealed \\
\hline & & K1CC: Contact fails closed & $\begin{array}{l}\text { Circuit remains energized but can } \\
\text { be broken by } \mathrm{K} 2\end{array}$ & Unrevealed \\
\hline & & K1CO: Contact fails open & No power to circuit & Revealed \\
\hline \multirow[t]{3}{*}{ Relay K2 } & \multirow[t]{3}{*}{ Delivers power to the motor } & K2D: Relay fails de-energized & No power to motor & Revealed \\
\hline & & K2CC: Contact fails closed & Continuous power to motor & Revealed \\
\hline & & K2CO: Contact fails open & No power to motor & Revealed \\
\hline \multirow[t]{2}{*}{ Timer relay (TIM) } & \multirow[t]{2}{*}{$\begin{array}{l}\text { Provides emergency shutdown in } \\
\text { event of pressure switch failing }\end{array}$} & $\begin{array}{l}\text { TIMCC: Timer contact fails } \\
\text { closed }\end{array}$ & $\begin{array}{l}\text { Circuit energized but PRSW can } \\
\text { open }\end{array}$ & Unrevealed \\
\hline & & $\begin{array}{l}\text { TIMCO: Timer contact fails } \\
\text { open }\end{array}$ & No power to motor & Revealed \\
\hline \multirow[t]{2}{*}{ Pressure switch (PRSW) } & \multirow{2}{*}{$\begin{array}{l}\text { De-energizes coil of } \mathrm{K} 2 \text { when tank } \\
\text { is full }\end{array}$} & PSWC: Fails closed & Continuous power to motor & Revealed \\
\hline & & PSWO: Fails open & No power to motor & Revealed \\
\hline Fuse & To prevent power surge & F: Fails broken & No power to motor & Revealed \\
\hline Power supplies 1 and 2 & Supplies power to relays and motor & PS1, PS2: No power & No power to motor & Revealed \\
\hline Motor & Pumps fluid into tank & M: Fails broken & No power to pump & Revealed \\
\hline
\end{tabular}




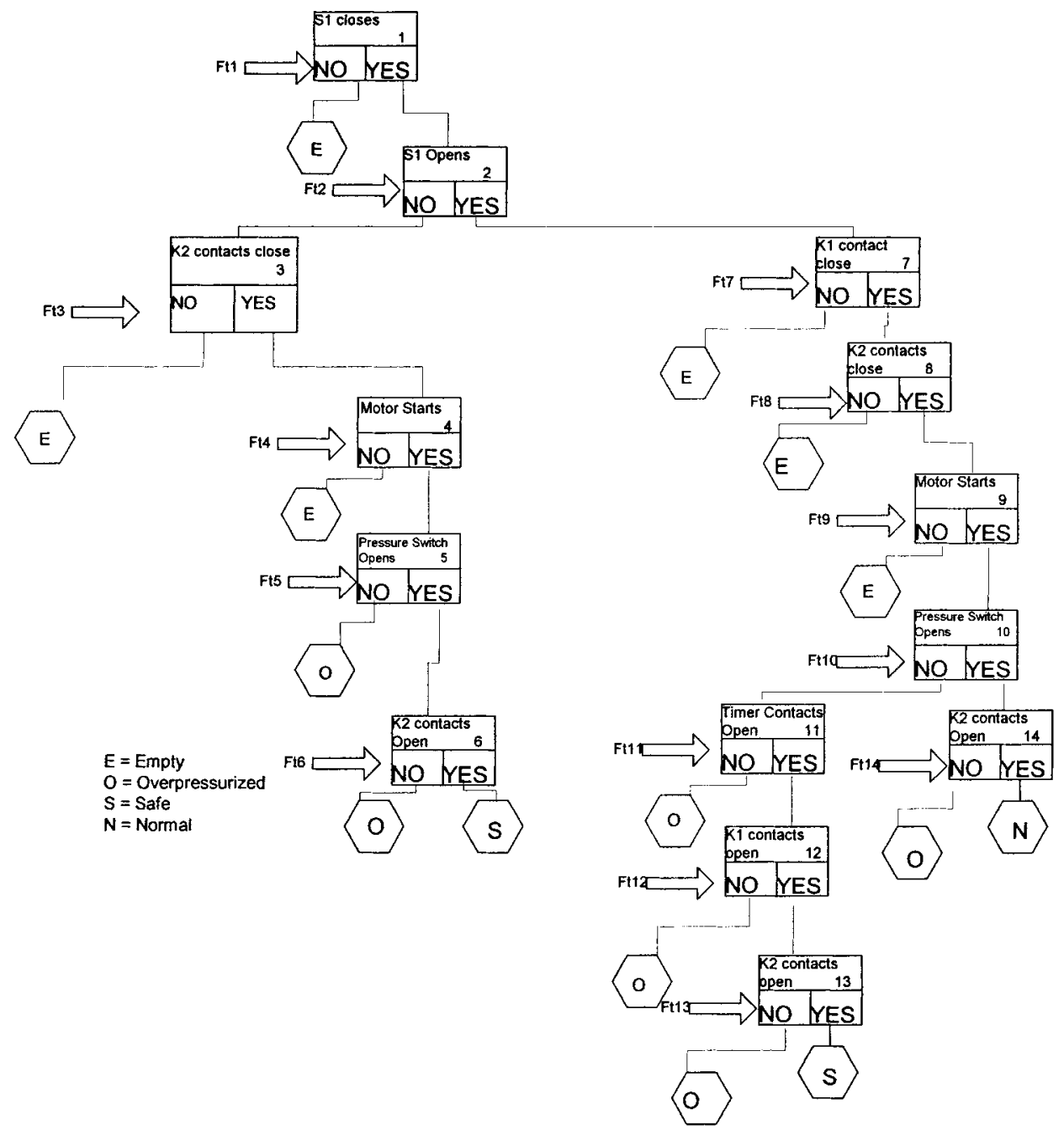

Fig. 9 Cause-consequence diagram for the pressure tank system

ing power from the pump. Where the timer is required to break the circuit containing $\mathrm{K} 1, \mathrm{~K} 1$ contacts should de-energize, removing power from $\mathrm{K} 2$, which results in the removal of the power supply to the pump. The ordering was therefore chosen to be

\section{$\mathrm{S} 1, \mathrm{~K} 1, \mathrm{~K} 2$, pressure switch, timer relay, K1, K2}

It can be seen that the components $\mathrm{K} 1$ and $\mathrm{K} 2$ both occur twice in the ordering sequence. This is the result of the system containing two different phases, and hence some components perform different actions in each different phase. The components $\mathrm{K} 1$ and $\mathrm{K} 2$ are both required to close in the start-up sequence and open in the shutdown sequence.

Steps 2 and 3. Cause-consequence diagram construction and reduction. The cause-consequence diagram was constructed by considering the effect of each component in the chosen order on the system performance. In order to highlight relevant features, only one filling sequence is investigated, the cause-consequence dia- gram of which is given in Fig. 9. The corresponding fault trees are illustrated in Fig. 10.

\section{SYSTEM QUANTIFICATION}

Prior to multiplying the probabilities associated with each decision box in each sequence, the cause-consequence diagram was checked for any dependent failure events. The following dependent failure events were identified*

1. Inconsistent failure event present in Ft1 and Ft2 as the switch is required to close, represented by decision box 1 , and then open, represented by decision box 2 . The second failure event, SIFC, is an unrevealed failure

\footnotetext{
* Following each of the seven modifications outlined, the causeconsequence diagram may change. These changes result in the duplication of certain parts of the diagram that may contain the inconsistent failures mentioned; e.g. the inconsistent failures detailed in 2 and 3 occur more than once and are handled in an identical manners.
} 


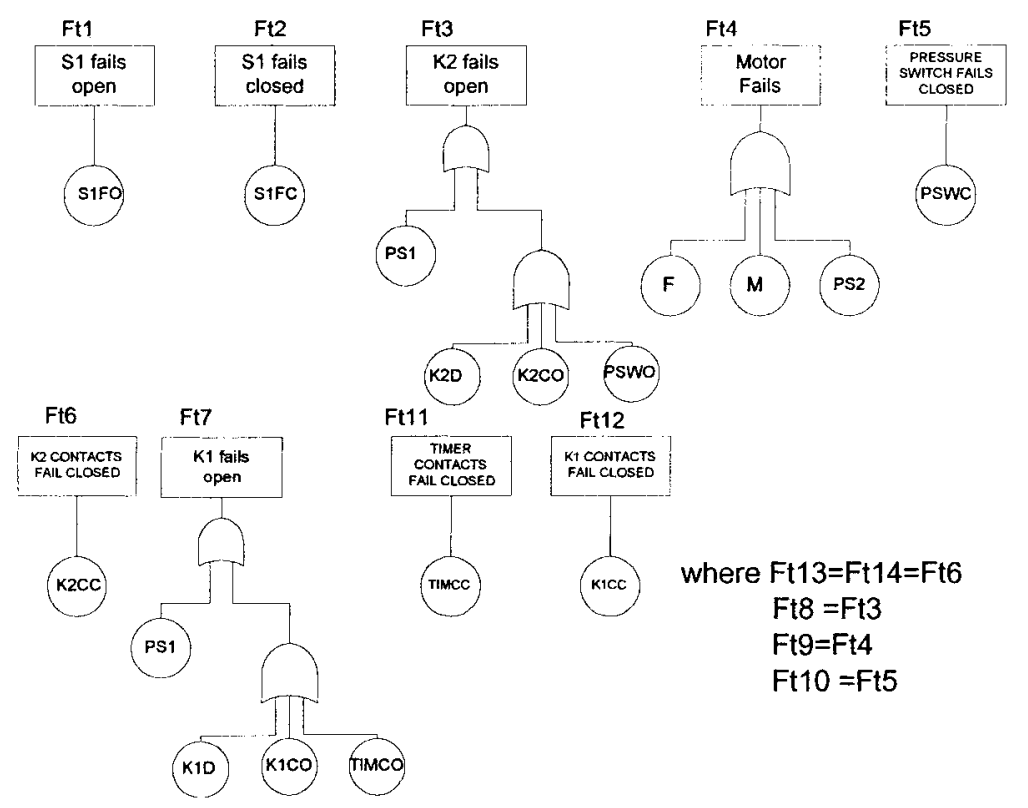

Fig. 10 Fault trees for the pressure tank cause-consequence diagram

event (Table 2) and is therefore extracted and placed in an existence decision box preceding decision box 1 . The cause-consequence diagram is modified using the procedure detailed in Section 5.1.2.

2. Inconsistent failure event present in Ft3 and Ft5 as the pressure switch is required to be closed and then open. The second failure event, PSWC, is a revealed failure event (Table 2) and the time to failure of PSWC is set equal to $30 \mathrm{~min}$ (the filling time).

3. Inconsistent failure event present in $\mathrm{Ft} 3$ and $\mathrm{Ft} 6$ as $\mathrm{K} 2$ contacts are required to close and, following the tank fill, open. The second failure event, $\mathrm{K} 2 \mathrm{CC}$, is a revealed failure event (Table 2) and the time to failure of $\mathrm{K} 2 \mathrm{CC}$ is set equal to $30 \mathrm{~min}$ (the filling time).

4. Common failure event present in Ft7 and Ft8. PS1 is extracted and placed in a new decision box preceding decision box 7 . The cause-consequence diagram is modified following the procedure detailed in Section 5.1.1.

5. Inconsistent failure event present in Ft7 and Ft12 as $\mathrm{K} 1$ contacts are required to close and then open. The second failure event, $\mathrm{K} 1 \mathrm{CC}$, is an unrevealed failure event (Table 2) and is therefore extracted and placed in an existence decision box. The cause-consequence diagram is modified using the procedure detailed in Section 5.1.2.

6. Inconsistent failure event present in Ft7 and Ft11 as the timer contacts are closed and may be required to open later in the sequence. The second failure event, TIMCC, is an unrevealed failure event (Table 2) and is therefore extracted and placed in an existence decision box. The cause-consequence diagram is modified using the procedure detailed in Section 5.1.2.

Following the appropriate modification owing to the dependent failure events identified, the final causeconsequence diagram was developed and is shown in Figs 11 and 12, with the corresponding fault trees given in Fig. 13. The probability of the system entering an overpressurized state was obtained using the component failure data shown in Table 3.

The system functions twice daily and therefore the time between operations is $12 \mathrm{~h}$. The probability of failure for revealed failures between operations was hence obtained using equation (1) with $t=12 \mathrm{~h}$. For unrevealed failures the probability of failure was obtained using $\theta$ and $\tau$, given in Table 3 , and equation (2):

$$
\begin{aligned}
& Q=1-\mathrm{e}^{-\lambda t} \\
& Q_{\mathrm{AV}}=\lambda\left(\frac{\theta}{2}+\tau\right)
\end{aligned}
$$

The probability of each fault tree was calculated using the inclusion-exclusion method (7), and the probability of overpressure was obtained by summing the probabilities of any sequence that terminated in the consequence ' $O$ '. There existed 12 such paths, and the probability of overpressure was calculated to equal $1.12 \times 10^{-5}$. In addition to obtaining the probability of overpressure, the probability of the tank being empty, a safe operation and a normal operation can also be calculated and shown to equal

$$
\begin{aligned}
& P(\text { normal operation })=0.766 \\
& P(\text { safe operation })=0.2213 \\
& P(\text { empty tank })=1.21 \times 10^{-2}
\end{aligned}
$$




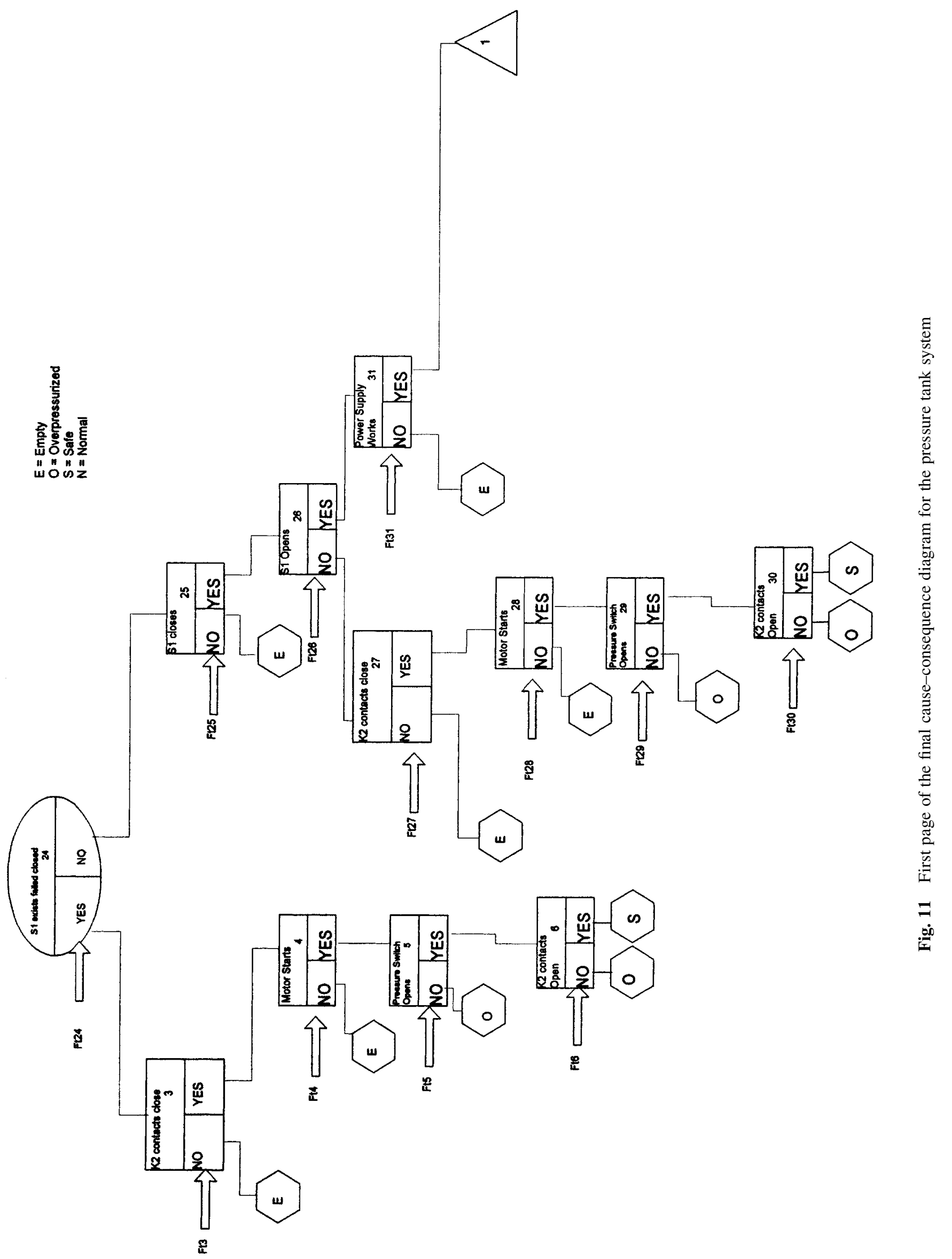




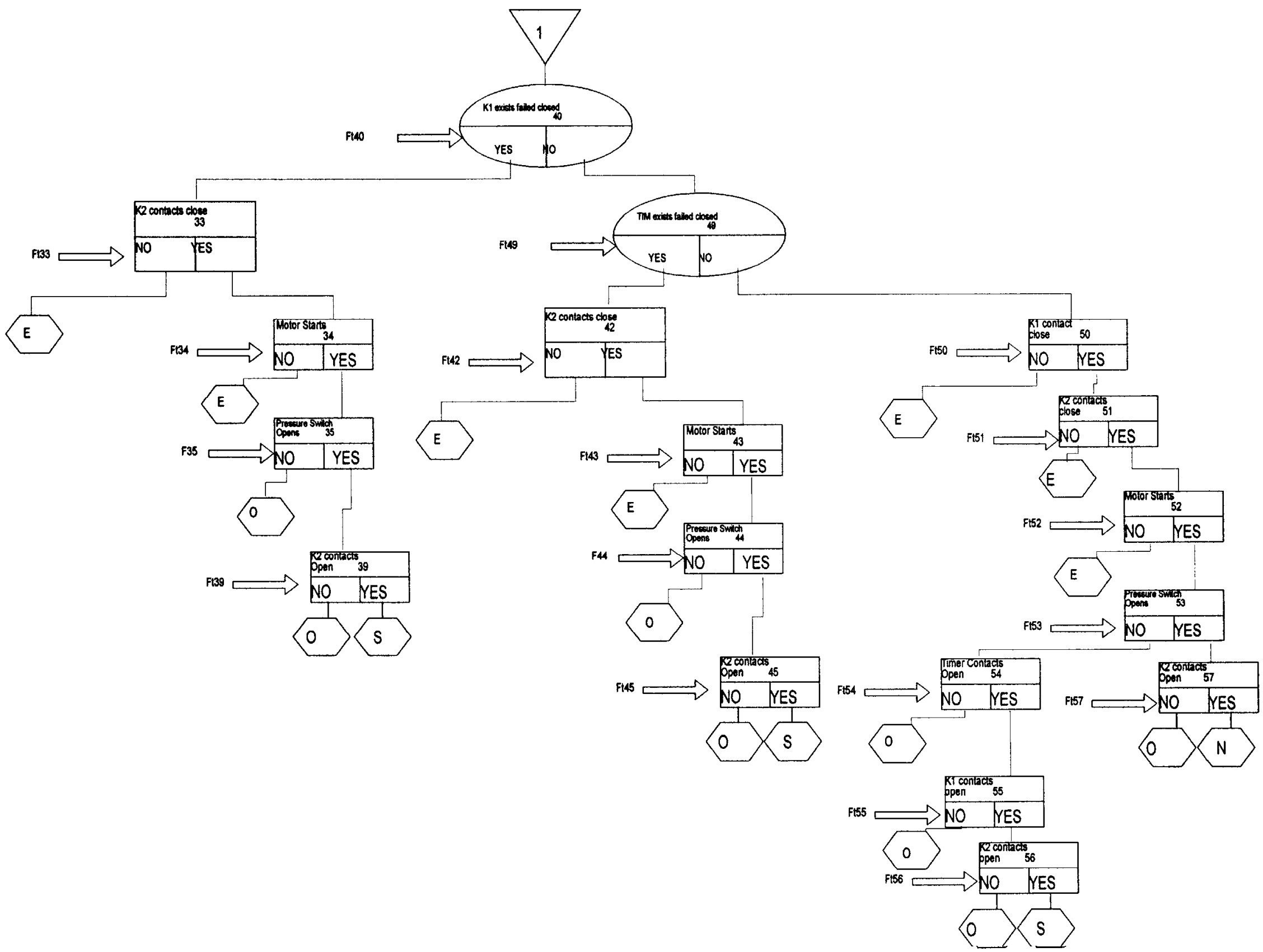




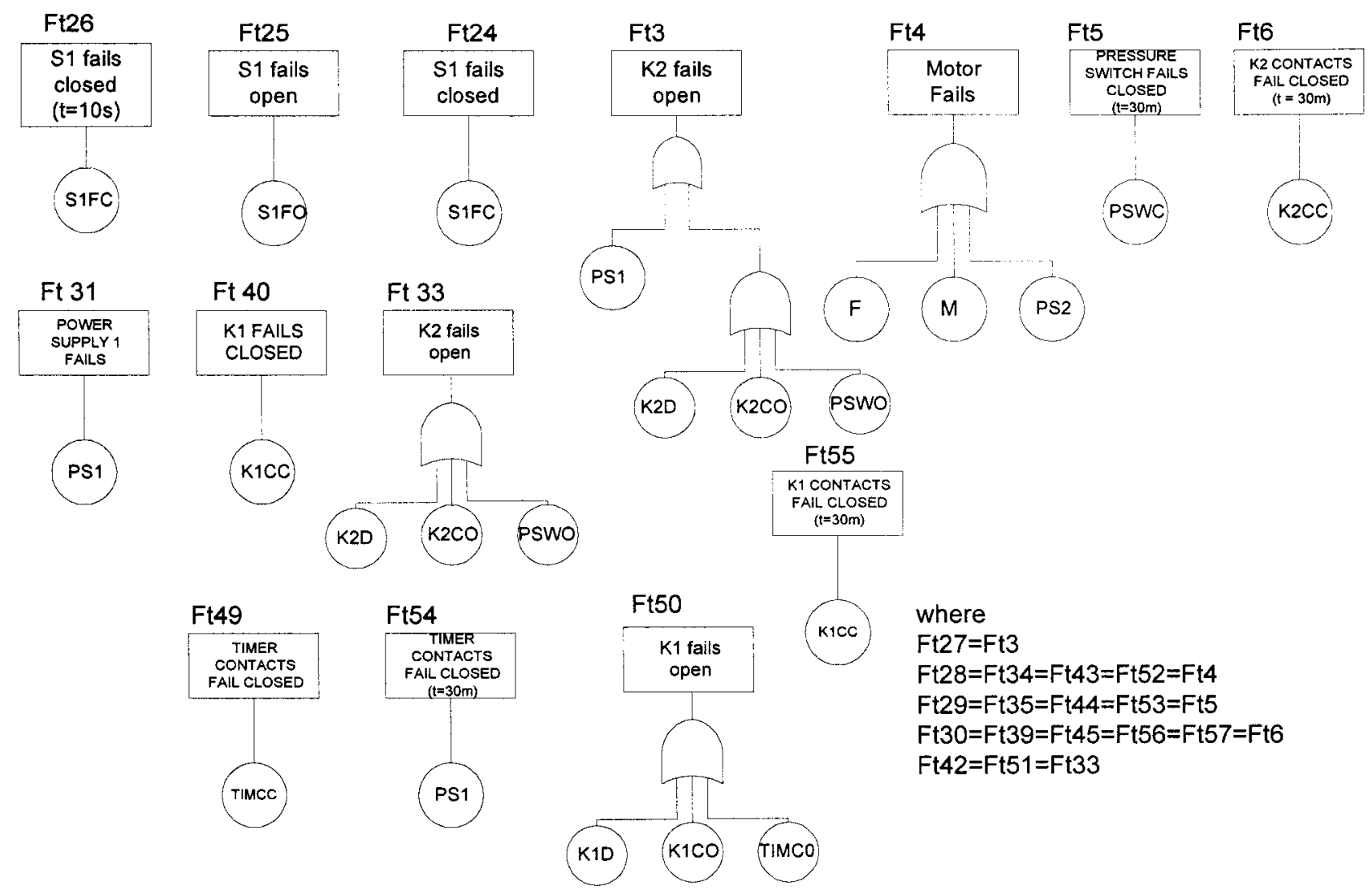

Fig. 13 Fault tree structures for Figs 11 and 12

\section{CONCLUSIONS}

The main advantage of the FTA method is that the failure logic of a system is well documented on the fault tree structure. Conventional fault tree quantification, however, requires a number of assumptions, which renders the analysis of sequential or dependent systems inaccurate. For such systems, an accurate analysis can be obtained via a Markov model, but the state transition diagram used in the Markov analysis holds no textual description regarding the failure logic of the system.

The cause-consequence diagram method enables

Table 3 Failure data for pressure tank system

\begin{tabular}{llll}
\hline Component & Failure rate & Inspection interval, $\theta$ & Mean time to repair, $\tau$ \\
\hline Switch S1 & S1FC: $1 \times 10^{-6}$ & 4368.0 & 36.0 \\
& S1FO: $8.698 \times 10^{-4}$ & NA & NA \\
Relay K1 & K1D: $0.23 \times 10^{-6}$ & NA & NA \\
& K1CC: $0.23 \times 10^{-6}$ & 4368.0 & 36.0 \\
Kelay K2 & K1CO: $0.23 \times 10^{-6}$ & NA & NA \\
& K2D: $0.23 \times 10^{-6}$ & NA & NA \\
K2CC: $0.23 \times 10^{-6}$ & NA & NA \\
Timer relay & K2CO: $0.23 \times 10^{-6}$ & NA & NA \\
Pressure switch & TCC: $1 \times 10^{-4}$ & 4368.0 & 36.0 \\
& TCO: $1 \times 10^{-4}$ & NA & NA \\
Fuse & PSWC: $1 \times 10^{-4}$ & NA & NA \\
Power supplies 1 and 2 & PSWO: $1 \times 10^{-4}$ & NA & NA \\
& F: $1 \times 10^{-5}:$ & NA & NA \\
Motor & PS1: $1 \times 10^{-6}$ & NA & NA \\
& PS2: $1 \times 10^{-6}$ & NA & NA \\
& & NA & NA \\
\hline
\end{tabular}


sequential or dependent systems to be modelled accurately with the retention of the failure logic for the system. In addition to this, more than one consequence can be modelled at a time as the cause-consequence diagram documents all system outcomes from a given critical event. In order to extend the capabilities of the cause-consequence diagram method, a list of construction and quantification rules have been developed and illustrated using an example system. In particular, this paper provides more rigorous definitions of the symbols used and the approach to be adopted to construct the cause-consequence diagram. A new symbol is introduced to distinguish between events that exist at a specified time and those that occur at that time. Once the cause-consequence diagram is constructed, its quantification can be complicated by dependencies between the events represented by the decision boxes. An approach to resolving this problem is given that can be automated within a computational analysis methodology. Dependencies attributed to either repeated events or inconsistent events can be accounted for in this way.

\section{REFERENCES}

1 Nielsen, D. S. The cause/consequence diagram method as a basis for quantitative accident analysis. Danish Atomic Energy Commission, RISO-M-1374, May 1971.

2 Andrews, J. D. and Moss, T. R. Reliability and Risk Assessment, 1993 (Longmans).

3 Billington, R. and Allan, R. Reliability Evaluation of Engineering Systems, 1983 (Pitman, London).

4 Villemeur, A. Reliability, Availability, Maintainability and Safety Assessment, 1991 (John Wiley, Chichester).

5 Nielsen, D. S. and Runge, B. Unreliability of a standby system with repair and imperfect switching. IEEE Trans. On Reliability, April 1974, 23, 17-24.

6 Taylor, J. R. Interlock design using fault tree analysis and cause-consequence analysis. RISO-M-1890, 1977.

7 Nielsen, D. S., Platz, O. and Runge, B. A causeconsequence chart of a redundant protection system. IEEE Trans. On Reliability, April 1975, 24(1).

8 Nielsen, D. S., Platz, O. and Kongs, H. E. Reliability analysis of proposed instrument air system. RISO-M-1903, April 1977.

9 Nielsen, D. S. Use of cause-consequence charts in practical systems analysis. Reliability and Fault Tree Analysis, 1975, pp. 849-880 (SIAM).

10 Hickling, P. The use of cause-consequence diagrams for the reliability analysis of sequentially operating systems. British Gas Report, 1980.

11 Hassl, D. F., Roberts, N. H., Vesely, W. E. and Goldberg, F. F. Fault Tree Handbook, 1981, NUREG-0492 (US Nuclear Regulatory Commission). 